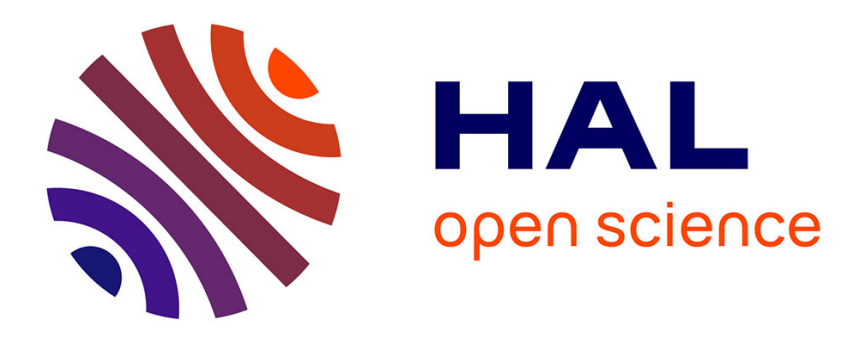

\title{
Remote PECVD : a Route to Controllable Plasma Deposition
}

\author{
S. Alexandrov
}

\section{To cite this version:}

S. Alexandrov. Remote PECVD : a Route to Controllable Plasma Deposition. Journal de Physique IV Proceedings, 1995, 05 (C5), pp.C5-567-C5-582. 10.1051/jphyscol:1995568 • jpa-00253930

\section{HAL Id: jpa-00253930 https://hal.science/jpa-00253930}

Submitted on 1 Jan 1995

HAL is a multi-disciplinary open access archive for the deposit and dissemination of scientific research documents, whether they are published or not. The documents may come from teaching and research institutions in France or abroad, or from public or private research centers.
L'archive ouverte pluridisciplinaire HAL, est destinée au dépôt et à la diffusion de documents scientifiques de niveau recherche, publiés ou non, émanant des établissements d'enseignement et de recherche français ou étrangers, des laboratoires publics ou privés. 


\title{
Remote PECVD: a Route to Controllable Plasma Deposition
}

\author{
S.E. Alexandrov \\ Department of Electronic Material Technology, St.-Petersburg State Technical University, Polytechnical \\ Str. 29, St.-Petersburg 195 251, Russia
}

\begin{abstract}
Some aspects of RF remote plasma enhanced CVD including the main features of the technique, possibilities of overcoming disadvantages typical for conventional plasma processes, and possible directions to improve the remote plasma method are discussed.
\end{abstract}

\section{INTRODUCTION}

Among a number of different low temperature deposition methods for various types of dielectric materials, the most widely used commercial method and most intensively studied process is that of plasma enhanced chemical vapour deposition (PECVD) [1]. The majority of PECVD systems have been based on the use of RF capacitively coupled parallel plate electrodes with a glow discharge in an appropriate gas mixture being formed between them. The substrates are usually placed on the surface of one of the electrodes. This conventional PECVD approach has several disadvantages, the main ones being :

- electrically active defects are likely to be generated near the surface of the semiconductor substrate due to bombardment by energetic particles during the deposition process;

- plasma bombardment of the growing dielectric film may cause a generation of pinholes and the appearance of charge in the film;

- complex reactions can be induced due to the simultaneous production of many different reacting species, some of which can participate in the film formation process leading to the deposition of films with unpredictable composition;

- process parameters such as the total pressure, RF-power, gas composition, gas flow rate and others are all interdependent and difficult to individually control.

Much of the research in the field of PECVD of dielectric films of various materials, and in particular silicon nitride and oxide, has been directed towards overcoming some of these problems [2]. One approach is to generate the plasma away from the substrate, so that there is less chance of plasma bombardment of the growing film. However, the advantages of this technique are still not completely clear in spite of considerable research which has been carried out $[2,3]$.

The aim of this paper is to focus on some aspects of RF remote PECVD including the main features of the technique, the real possibility of overcoming the above mentioned disadvantages, and the possible improvement of the remote plasma method by the excitation of noble gas atoms into metastable excited electronic states and their use for the generation of the active species in the 
gas phase required for film formation. These points will be discussed using the example of remote PECVD of silicon nitride films, which are attracting considerable interest as excellent dielectric layers [4].

\section{REMOTE PLASMA ENHANCED CVD : ADVANTAGES AND DISADVANTAGES}

Extensive studies of remote RF PECVD have been carried out since 1978, when the remote plasma process was described by Helix [5] for the first time. Approximately 40 papers, mainly related to deposition of silicon based materials have been published. Most of these were reviewed by Lucovsky et al [3].

According to Lucovsky, who has made extensive studies of remote plasma processes, there are two main features of these kind of processes: (1) the substrate must be placed outside of the plasma generation region and (2) "not all of the process gases are subjected to direct plasma excitation" [3]. In accordance with these features there are at least two types of modifications for the remote PECVD processes depending on which of the methods of RF power coupling into the reaction chamber is used. In the majority of remote processes, RF power is coupled into the system inductively for excitation of some of initial reagents (see Figure 1). A schematic diagram of the experimental apparatus with capacitively coupled RF power, which was developed two years ago for formation of silicon nitride films [6], is shown in Figure 2. As one can see in both reaction chambers the plasma generation regions and the areas of reactors used for substrate location are separated in space. Only some of the initial reagents are excited by direct collisions with accelerated electrons in the plasma generation regions, and these are then transported in the direction of the deposition zone. Other reactants are introduced downstream of the discharge generation region and can undergo indirect excitation.

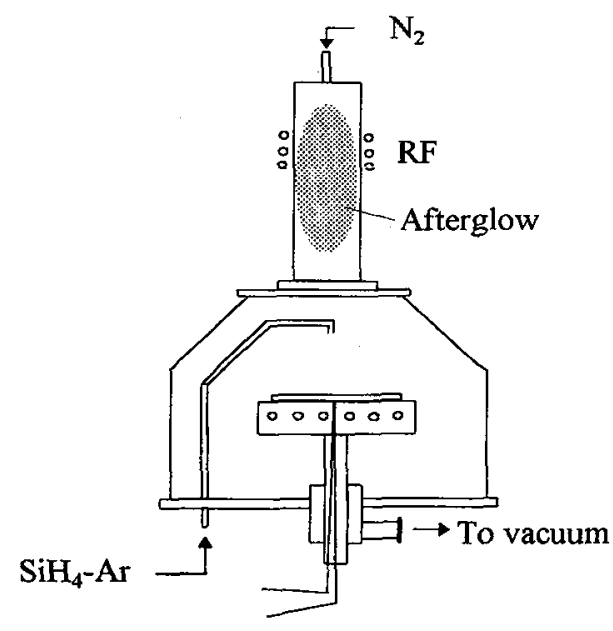

Figure 1: Schmatic diagram of the remote PECVD reactor with inductively coupled RF power.

The results of experiments of remote PECVD of silicon nitride films show that layers with nearstoichiometric composition, formed on various substrates and using both modifications of the process, contain relatively low concentration of bonded hydrogen $\left(3 \cdot 10^{21} \mathrm{~cm}^{-3}[7]\right.$ and $9 \cdot 10^{21} \mathrm{~cm}^{-3}$ 
[8]), have good dielectric and insulator properties, and can be successfully used for electronic applications. However, the information that has been published relating to the development of remote PECVD has not really brought out the advantages of this technique from the point of view of overcoming the above mentioned disadvantages of conventional plasma deposition processes,



Figure 2: Schematic diagram of the remote PECVD reactor with capacitively coupled RF power.

although the technique has been suggested as providing a solution to the problem concerning a minimisation of the density of electrically active defects generated near the semiconductor surface due to bombardment by energetic particles during deposition. To confirm this, we have deposited various dielectric materials on the surface of special test gateless GaAs structures (see Figure 3) using different deposition techniques. Comparing the values of saturation current (the maximum possible current in the top layer) obtained from the static I-V curves of these structures before and after deposition, it is possible to evaluate the influence of plasma bombardment on the surface properties of the semiconductor. To prevent possible influence of electric charge, which can be accumulated in the growing dielectric films during deposition, the deposited layers were chemically etched before measurements. Because of the relatively low mean electron energy (1-3 eV [2]) typical for low pressure RF PECVD, the sputtering of the channel of the structure (the area of top layer placed between ohmic contact patterns) would not be expected. Generation of defects near a semiconductor surface will cause a decrease of saturation current because of a decrease of effective thickness in the above layer. The results of these experiments are shown in the Table 1 . It should be noted that in all cases the dielectric deposition processes did not influence the general appearance of the I-V curves, but it can be seen from the data contained in the Table 1 that the values of saturation current of the structures were decreased $30-40 \%$ after deposition of both $\mathrm{SiO}_{2}$ and $\mathrm{SiN}_{\mathrm{x}} \mathrm{H}_{\mathrm{y}}$ films by conventional PECVD. The saturation current was not affected by low pressure thermal CVD of $\mathrm{GaO}_{\mathrm{x}} \mathrm{N}_{\mathrm{y}}$ films (without plasma excitation) or by remote PECVD of $\mathrm{SiN}_{\mathrm{x}} \mathrm{H}_{\mathrm{y}}$ films. These results indicate that the surface of a semiconductor undergoes much less degradation during the remote plasma processes when compared with conventional plasma methods. This considerable advantage 
of remote PECVD is emphasised in its industrial application. It should be noted that this conclusion is also relevant to plasma etching processes as well.

Table 1. Values of the saturation current of the gateless GaAs structures before and after deposition of various dielectric films by different deposition methods.

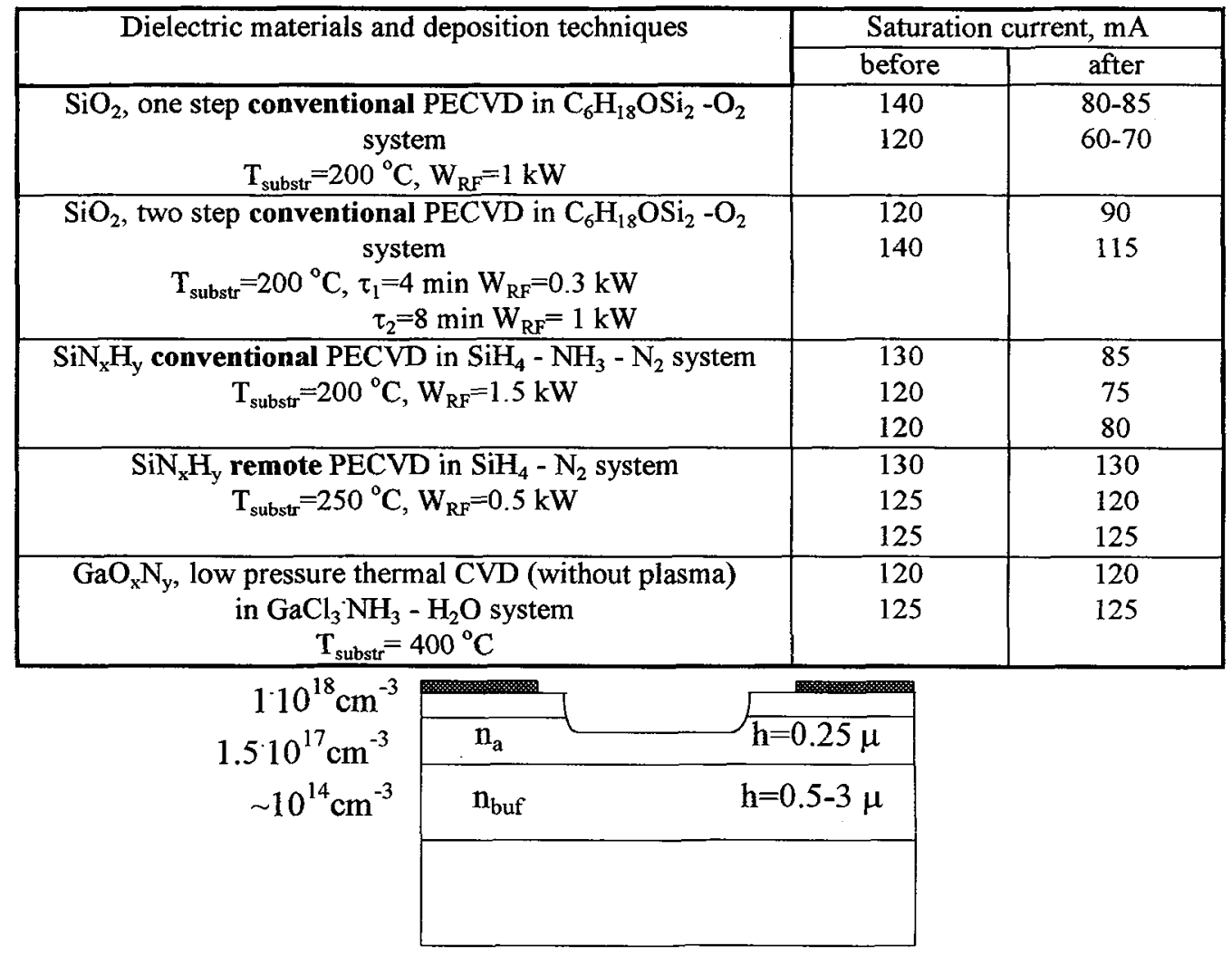

Figure 3: The test gateless GaAs structure.

Less interdependence of the control of process parameters should also be expected in the case of remote PECVD processes, although it is not expected to be completely eliminated. During conventional PECVD of silicon nitride films in $\mathrm{SiH}_{4}-\mathrm{N}_{2}$ system even small variations of silane flow rate cause dramatic changes in the plasma phase composition because of the dissociation of silane, and the formation of various types of radicals and atoms, such as $\mathrm{SiH}_{3}, \mathrm{SiH}_{2}, \mathrm{SiH}, \mathrm{Si}$, and H [9]. These changes of plasma compositions occur directly in the discharge generation region, and therefore this leads to variations of the level of RF power dissipated in the plasma. In the case of a remote plasma process, when silane is introduced downstream from the discharge region and with gas flow conditions to minimise or completely prevent backstreaming of silane in the direction of the discharge generation zone, the variation of $\mathrm{SiH}_{4}$ flow rate does not cause variation of $\mathrm{RF}$ power absorbed in the plasma. Another example of the lower interdependence of the process parameters during remote plasma deposition can be seen when regarding the process occurring on the surface 
of the substrate and growing film. It is known [1], that real temperature of the reaction surface is determined not only by the heat flux from susceptor but also by the flux of energy delivered to the reaction surface by energetic particles from the plasma. Variations in the level of RF power absorbed in the plasma (such as those discussed above) must cause the changes of reaction surface temperature for conventional PECVD. It is clear that this interdependence would be expected to be much less in the case of remote plasma deposition. However, it should be noted that some interdependence of process parameters still occurs during remote PECVD, and further improvements cannot be readily made. For example, variations in the total pressure in both remote and conventional PECVD lead to dramatic changes in RF power absorbed in the plasma, which effects the plasma phase composition.

These considerations, nevertheless, allow us to conclude that remote PECVD provides less interdependence for some process parameters, and so it can be more readily controlled. However, completely overcoming this problem for any low pressure RP PECVD is impossible.

Another disadvantage of conventional PECVD, mentioned above, is that complex reactions can occur as a result of the simultaneous production of different reacting species in the plasma phase when inelastic collisions occur with accelerated electrons of various energies. It is well known that the electron energy distribution function for this type of RF glow discharge is satisfactory described by a Maxwellian distribution over the energy range 0.1-15 eV [10]. Typical mean values of electron energies are in the region 2-4 eV, but the fastest electrons can reach energies up to $30 \mathrm{eV} \mathrm{[11].}$ Inelastic collisions of these electrons with molecules of precursor(s), directly introduced into the discharge generation region, cause the simultaneous production of different reacting species, in accordance with the electronic structure of precursor molecules and electron energy distribution, which are dependent on process parameters. It is clear that, although this problem will be less marked than in the case of conventional PECVD, because only the less reactive gas species are generally excited, it can not be completely overcome because of the nature of a glow discharge, created by subjecting the initial gases to an electromagnetic RF field.

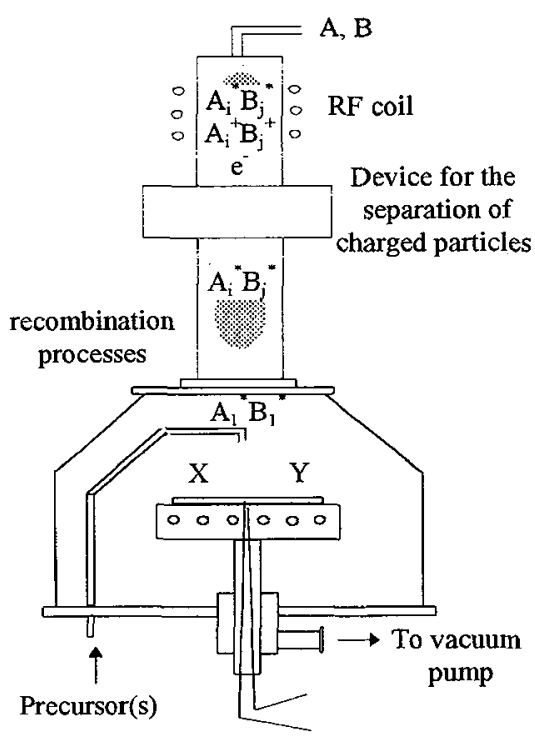

A



B

Figure 4: Schematic diagrams of the "ideal" processes of remote PECVD 
In the remote plasma deposition processes, charged particles (accelerated electrons and ions) are likely to be easily transported by the gas flow from the discharge generation region to the point of mixing of the initial reagents [3]. These charged particles are characterised by a continuous electron energy distribution over a wide range, and the above mentioned formation of different reacting species takes place in the region of mixing due to collisions of molecules of precursors with the charged particles. These active species, then transported to the deposition zone, may participate in the film formation process as well as in ancillary homogeneous reactions. This mechanism leads to the deposition of films with unpredictable composition. Thus, the third important difficulty associated with conventional PECVD deposition, is unresolved by remote PECVD.

This problem can be solved, though, when the penetration of charged particles downstream from the plasma generation region in the direction of the point of mixing and the deposition zone is stopped. In this case, formation of the active species required for film growth can occur both in the discharge generation region, and by collisions of the precursor molecules introduced downstream from the plasma region, with neutral excited particles extracted from the discharge area. These neutral particles have definite energies which in the ideal case must coincide with the values of energy necessary for the selective production of reacting species. The separation of charged from neutral particles can be achieved by using electric or magnetic fields. Thus, in an improved method the discharge generation region is used as a source of only neutral particles with the necessary discrete energies. Schematic presentations of the "ideal" processes of remote PECVD deposition are shown in figures $4 \mathrm{a}, \mathrm{b}$.

It should be noted that neutral particles extracted from the discharge region have various energies, over a wide range, corresponding to their definite electronic excited states. These excited particles can be separated because of differences in life times. In the gas stream which moves from the discharge region in the direction of the deposition zone along the reactor, the particles with relatively low life times recombine first, and the long-lived particles will move on. Taking this into account an appropriate location of the point of mixing of the precursor molecules with the neutral particles, to be used for excitation, can be chosen. If two different precursors are to be used for the film deposition process, and the neutral particles with an energy necessary for the excitation of one of the precursor molecules cause undesirable dissociations or excitations of the molecules of the other precursor, a reaction chamber with two separate zones of plasma excitation should be used (see Fig. 4b).

From the above discussion it can be seen that, although there may still be some drawbacks, remote plasma CVD looks quite promising for overcoming some of the disadvantages of conventional PECVD. One point which should be mentioned is that there is a risk of generating electrons due to recombination of excited neutral particles, via Penning type interaction in the area of the reactor downstream from the device used for the separation of charged particles. The electrons formed by this mechanism usually have low energies and in the absence of electric and magnetic fields are not able to increase their energies. Hence they can only enhance processes of excitation, dissociation or ionisation with relatively low threshold energies; for example, many reactions involving silane are characterised with low energies in the range $2-5 \mathrm{eV}$ [9]. In many other cases, however, the energy of these electrons will not be enough to participate in the above mentioned processes.

Remote plasma processes, as described above, are simpler than direct PECVD but will still have complicated mechanism, including several consequentive steps. A deeper understanding of some of these steps is likely to be useful.

One of the most interesting points which should be discussed in connection with the development of remote plasma processes is that concerning the nature of particles which can be used for excitation of the precursor molecules. These particles should have considerable life times, 
should be chemically inert with respect to the other reagents and reactor materials, and must have energies which, in the ideal case, must coincide with the energy required for the selective production of reacting species.

Atoms of noble gases such as $\mathrm{He}, \mathrm{Ar}$, as well as stable molecules of other gases (for instance, nitrogen), in metastable electronic states, with considerable radiative life times, are likely to be successfully used as neutral particles for transferring energy to the molecules of precursors. In spite of a considerable number of papers dedicated to the use of noble gases for an improvement of plasma deposition, their role during plasma enhanced CVD processes is still not clear. The possibility of formation of high-energy atoms of noble gases being in metastable long-lived electronic states is a typical feature of these processes. The excited atoms of noble gases are capable of exciting and ionising particles of other reagents being used for deposition. The results of a study of the influence of helium dilution on the remote PECVD of layers in the $\mathrm{SiH}_{4}-\mathrm{N}_{2}$ and $\mathrm{SiH}_{4}-\mathrm{O}_{2}$ systems obtained by Tsu and co-authors [12], show that at high partial pressures of $\mathrm{N}_{2}$ and $\mathrm{O}_{2}$ helium acts as a diluent only, while at low partial pressures metastable helium atoms are responsible for the dissociation of silane and an increase in the concentration of bounded hydrogen in the deposited films. It was also assumed that metastable helium does not affect the concentration of the active nitrogen atoms in the gas phase. The results obtained in studies of the growth of crystalline $\mathrm{Si}$ and Ge films[13] showed that metastable long-lived argon atoms can play an active role in the formation of intermediates, which are then responsible for the film growth. It is clear that the effect caused by the introduction of noble gases into the initial reaction mixture on PECVD depends on the experimental conditions under which the process is carried out and the nature of the initial reagents in each specific case.

For a better understanding of the role of the dilution of the reactant gas mixture with noble gases, such as Ar, during remote PECVD of silicon nitride films, and for the experimental examination of use of atoms of noble gases in metastable states for the selective excitation of the precursor molecules, an optical emission spectroscopic study has been carried out.

\section{EMUSSION SPECTROSCOPIC STUDY OF THE INFLUENCE OF ARGON DILUTION ON NITROGEN PLASMA PARAMETERS.}

A study was performed using the experimental setup described previously in detail [14] and presented schematically in Fig. 1. Nitrogen and argon were introduced into the quartz reaction chamber from the top of the reactor, and then passed through the RF discharge region in the direction of the susceptor by means of evacuation of the reactor from the bottom. RF power was inductively coupled into the system.

The analysis of a typical emission spectrum of the nitrogen-argon plasma, obtained from an area about $5 \mathrm{~cm}$ below the inductor coil allows us to distinguish several groups of bands belonging mainly to nitrogen (see Fig. 5). In each group, line intensity varies symbiotically with the total pressure and RF power. The emission lines in the region $290-450 \mathrm{~nm}$ were the most intensive and they constitute the "second positive" system of emission lines of nitrogen, corresponding to the transitions of molecular nitrogen from the $C^{3} \Pi_{u}$ excited electronic state $(v "=0-4)$ with $11.1 \mathrm{eV}$ energy $\left(v^{\prime}=0\right)$ to the low-lying $B^{3} \Pi_{g}$ excited state $\left(v^{\prime \prime}=0-7\right)$ with $7.4 \mathrm{eV}$ energy $\left(v^{\prime \prime}=0\right)$. The breaking of the sequence of bands corresponding to the second positive system of nitrogen at $v^{\prime}>4$ of the $C^{3} \Pi_{u}$ state is caused by predissociation. A group of lines in the region $200-270 \mathrm{~nm}$ are characterised by substantially (approximately 10 fold) lower relative intensities. This group constitutes the fourth positive system of bands attributed to the transitions of molecular nitrogen from the $D^{3} \Sigma_{u}{ }^{+}$excited state $\left(13 \mathrm{eV}\right.$ for $\left.v^{`}=0\right)$ to the $B^{3} \Pi_{\mathrm{g}}$ excited state. 

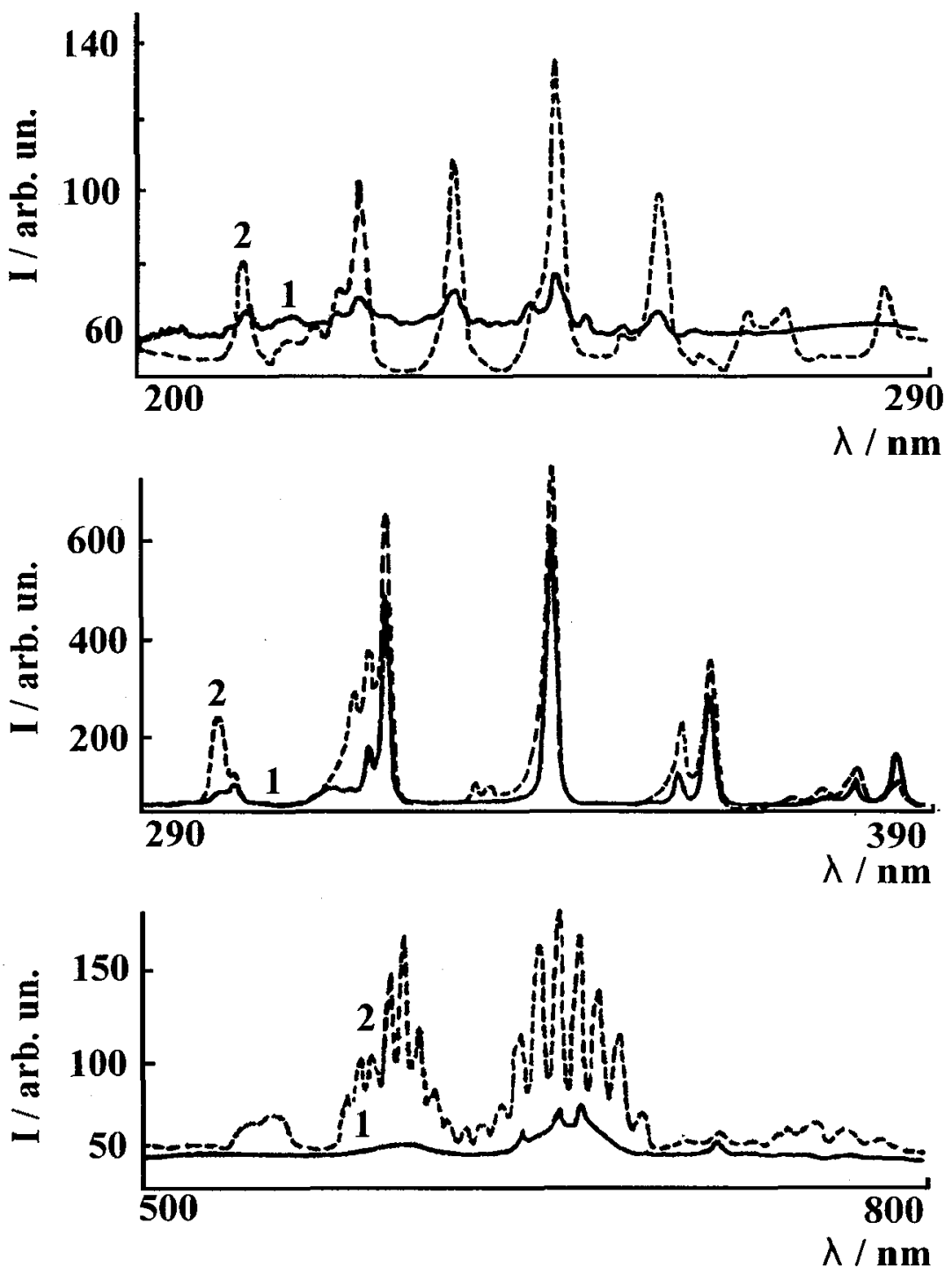

Figure 5: Emission spectra of nitrogen (1) and nitrogen-argon (2) plasma. $P_{\text {tot }}=200 \mathrm{~Pa}, \mathrm{~W}_{\mathrm{RF}}=100 \mathrm{~W}$ 
Apart from the emission lines of the second and fourth positive systems in the spectra studied, a group of lines, characterised by substantially lower intensities, was found in the region 500-800 nm. It was previously shown [14], these lines belong to the first positive system of bands, corresponding to the $\mathrm{B}^{3} \Pi_{\mathrm{g}} \rightarrow \mathrm{A}^{3} \Sigma_{\mathrm{u}}{ }^{+}$transition of molecular nitrogen, mainly from the upper vibrational levels $\left(v^{\prime}<13\right)$, which lie below the predissociation threshold for molecular nitrogen. The unexpected population of the latter states is caused by the reverse predissociation of nitrogen. The noted series of lines are typical of an afterglow spectra of a low pressure RF discharge nitrogen plasma and constituted the so-called Lewis-Rayleigh glow [15]. The anomalous increase in the intensity of these lines may occur only in the presence of atomic nitrogen in the ${ }^{4} S$ state in the plasma studied.

As well as lines attributed to the emitting transitions of molecular nitrogen, two lines at 451 and $433 \mathrm{~nm}$ were found in the spectra obtained, which were attributed to the transitions between excited electronic levels of argon atoms [16]. It should be noted, though, that their intensity (even at $90 \%$ Ar in the gas mixture) was comparable with the noise.

Direct electronic excitation of argon atoms to metastable states with 11.55 and $11.72 \mathrm{eV}$ energy [17] is unlikely, and transitions to these levels seem to proceed via excited states with energies at 13 $\mathrm{eV}$ and above. The transitions considered are emitting, and the corresponding emission lines, attributed to transitions from 14.57 and $14.68 \mathrm{eV}$ energy levels, were observed at 451 and $433 \mathrm{~nm}$, respectively; as already mentioned though, they were comparable with the noise in ccontrast with the lines of the second positive system of nitrogen. Nevertheless, the results obtained are evidence of the presence of argon atoms in metastable states, which can be involved in nitrogen excitation. In this connection it is of interest to mention that energies of argon atoms occurring in metastable states are close to those required for excitation of molecular nitrogen to certain vibrational levels of $\mathrm{C}^{3} \Pi_{\mathrm{u}}$ electronic state $\left(11.51 \mathrm{eV}\right.$ for $v^{`}=2$ and $11.74 \mathrm{eV}$ for $\left.v^{`}=3\right)$ and also are comparable with the energy of the ground vibrational level of the $C^{\prime \prime 5} \Pi_{u}$ electronic state of $N_{2}$ (11.6 eV[18]). Therefore, one can expect that nonelastic collisions of metastable argon atoms with $\mathrm{N}_{2}$ molecules will increase the population of the above mentioned vibrational levels, as well as increasing the concentration of ${ }^{4} \mathrm{~S}$ and ${ }^{4} \mathrm{~B}$ atomic nitrogen, formed either by the predissociation mechanism from the $\mathrm{C}^{3} \Pi_{\mathrm{u}}$ electronic state or by direct dissociation of a nitrogen molecule in the $C^{\prime}{ }^{\prime 5} \Pi_{u}$ state.

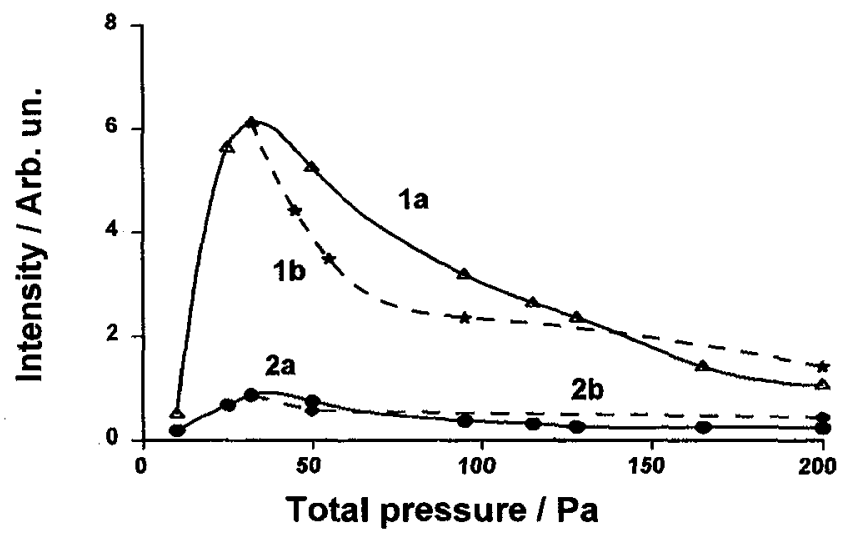

Figure 6: Integral intensity of emission lines of excited nitrogen molecules as a function of total pressure. a-pure nitrogen plasma; $b-A r-N_{2}$ mixture $\left(\mathrm{P}_{\mathrm{N} 2}{ }^{-} 35 \mathrm{~Pa}\right)$; 1 - "second" and 2 - "fourth" positive system. 
Figure 6 presents the integral (ie, summed over all lines) "intensity-total pressure" dependencies for the second and the fourth positive systems of molecular nitrogen both for a pure nitrogen plasma (curves $1 \mathrm{a}$ and $2 \mathrm{a}$ ) and a nitrogen-argon plasma (curves $1 \mathrm{~b}$ and $2 \mathrm{~b}$ ). In the latter case, the partial pressure of nitrogen was maintained constant $(35 \mathrm{~Pa})$, and the total pressure in the reactor was changed by dilution with argon. In the case of a pure nitrogen plasma, an increase in pressure of up to $35 \mathrm{~Pa}$ leads to an increase in the concentration of nitrogen molecules in the gas phase and, consequently, the fraction of nitrogen molecules in excited $C^{3} \Pi_{u}$ and $D^{3} \Sigma_{u}{ }^{+}$electronic states. This is particularly responsible for the increased intensity of corresponding emission lines, since it was shown previously that the mean electron temperature remains constant over the wide pressure range investigated (10-200 Pa), namely $0.24 \mathrm{eV}$ [14]. A further increase in pressure decreases the line intensity, apparently because of decreased electron concentration due to recombination processes.

In the case of an argon-nitrogen plasma, the appearance of the curve characterising the variation in integral intensity with pressure remains almost unchanged. When small amounts of argon were introduced into the gas phase $\left(\mathrm{P}_{\mathrm{Ar}} / \mathrm{P}_{\mathrm{N} 2}<1-2\right)$ the integral intensity of the second and the fourth positive systems of nitrogen proved to be slightly lower than those for the pure nitrogen plasma. This fact is likely to be caused by the lower electron concentration in the argon-nitrogen discharge, since argon is a harder to excite than nitrogen [16]. Another reason for a decrease in the emission intensity is the lower efficiency of nitrogen excitation with argon (at its low concentrations in the
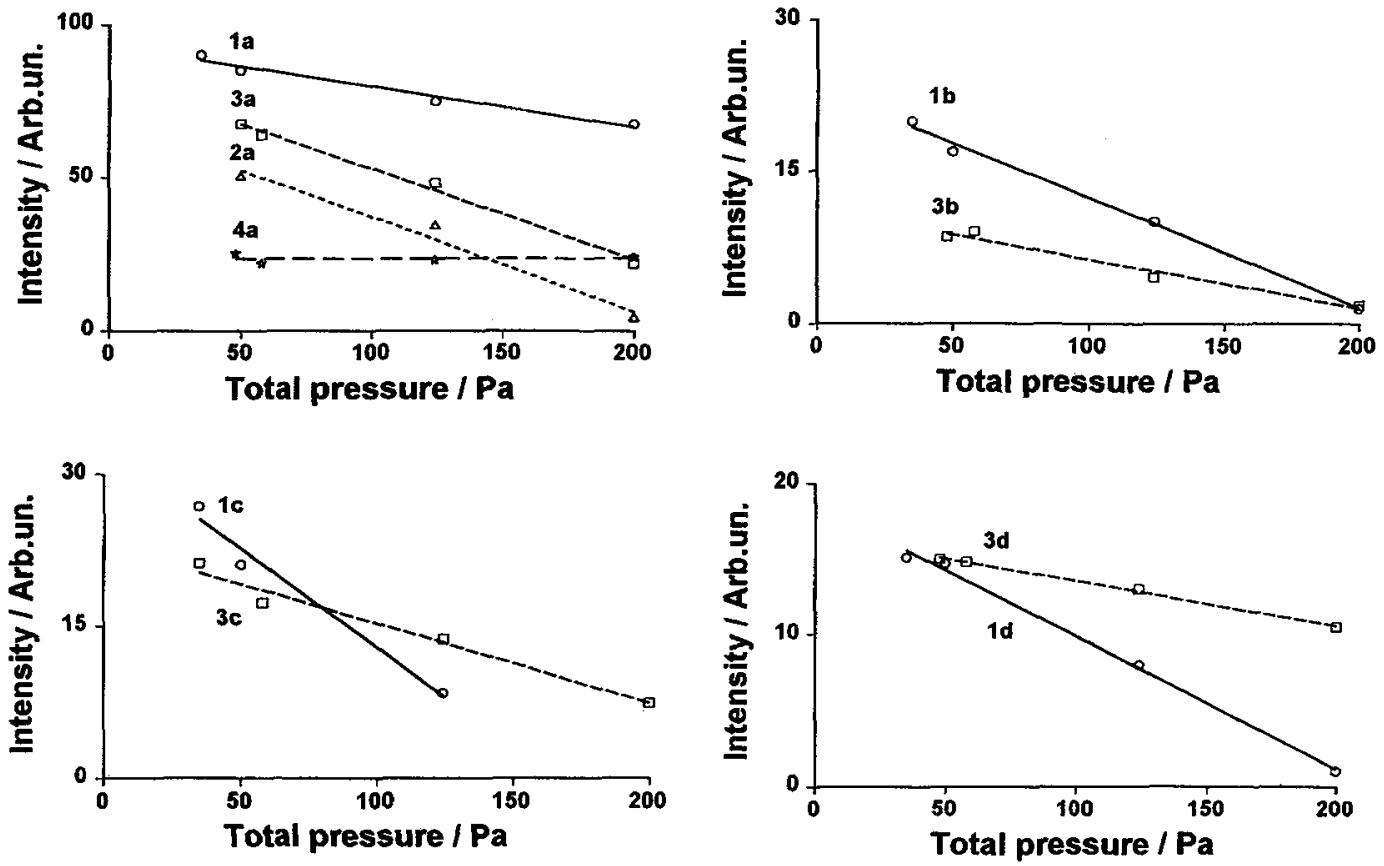

Figure 7: Integral intensity for the selected emission linesof the second and fourth positive systems of nitrogen; a$0 \rightarrow 0, b-2 \rightarrow 0, c-3 \rightarrow 2, d-4 \rightarrow 3$ transitions; $1-2$ nd and 2-4th for pure $\mathrm{N}_{2} ; 3-2$ nd 4-4th for $\mathrm{Ar}_{2} \mathrm{~N}_{2}(35 \mathrm{~Pa})$. 
gas phase) in comparison with the efficiency of electronic excitation. This is because of differences in particle size and velocity. A further increase in argon concentration in the initial gas mixture $\mathbf{P}_{\mathrm{Ar}} / \mathbf{P}_{\mathrm{N} 2}>3$ ) results in an increase in the intensities of lines belonging to the both systems in contrast with those in a nitrogen plasma. The most considerable (more than twofold) increase is observed for the lines of the fourth positive system. This effect is likely to be determined by the increased probability of nitrogen excitation with long-lived metastable argon atoms, which are capable of lower recombination at higher pressures as compared to electrons, and also with argon atoms which are in excited states with energies above $13 \mathrm{eV}$.

Figure 7 presents the "integral intensity-total pressure" dependencies for selected lines of the second and fourth positive systems. Analysis of these data allow us to understand better the role of argon in nitrogen excitation. Also, analogous curves for nitrogen are shown in the same figure for comparison. As can be seen, the pressure increase due to adding of argon leads to a monotonic decrease in the intensities of all lines. However, unlike the case of a pure nitrogen plasma, the rate of the fall off in the intensities with pressure depends on the vibrational quantum number $v$ of the $C^{3} \Pi_{u}$ state of molecular nitrogen, from which the emitting transitions occur, belonging to the second positive system of nitrogen. The intensity of the $0 \rightarrow 0$ line is always lower in the case of a nitrogen-argon plasma. A steeper decrease of the $2 \rightarrow 0$ line intensity in the case of a nitrogen-argon plasma leads to the equality of line intensities for both kinds of plasma at $P_{\mathrm{Ar}} / \mathbf{P}_{\mathrm{N} 2}=6$, the total pressure being the same. The $3 \rightarrow 2$ line intensity for nitrogen plasma exceeds that for nitrogenargon plasma only at small $\mathrm{P}_{\mathrm{A} r} / \mathrm{P}_{\mathrm{N} 2}$ ratios $(<1.5)$, whereas at higher partial pressures of argon the opposite relationship is observed. As far as the $4 \rightarrow 3$ line intensity is concerned, one can see that even small amounts of argon added to nitrogen cause it to increase in the case of a nitrogen-argon plasma in contrast with a nitrogen plasma for increasing $\mathrm{P}_{\mathrm{Ar}} / \mathbf{P}_{\mathrm{N} 2}$ ratio.

The regularities found in the variation of line intensity with pressure for the second and fourth positive systems of nitrogen can be explained by direct participation of argon atoms, being in metastable states, in nitrogen excitation to vibrational levels of the $C^{3} \Pi_{u}$ state, characterised by $v^{\prime}=2$ and 3 . However, the energy of metastable argon atoms is not sufficient for pumping of the vibrational state with $v^{`}=4$, belonging to the $C^{3} \Pi_{u}$ electronic state of molecular nitrogen $(12 \mathrm{eV})$. The anomalous population density of the latter state is most likely to be caused by reverse predissociation of nitrogen.

$$
N\left({ }^{4} S\right)+N\left({ }^{2} D\right)+M=C^{` 5} \Pi_{u}+M=C^{3} \Pi_{u}\left(v^{`}=4,3,2\right)
$$

It was mentioned previously that metastable argon atoms are close in energy to vibrational states belonging to the $C^{3} \Pi_{u}$ state of $N_{2}$. Hence, it seems likely that nonelastic collisions of nitrogen molecules with metastable argon atoms increase the probability of predissociation of molecular nitrogen both from the $C^{3} \Pi_{u}$ state directly and through the $C^{\prime \prime 5} \Pi_{u}$ electronic state of $N_{2}$, resulting in the formation of nitrogen atoms in ${ }^{4} \mathrm{~S}$ and ${ }^{2} \mathrm{D}$ states. This should be accompanied, however, by an increase of the probability of the reverse predissociation, when a fraction of excited molecules descend to the closest low-lying vibrational levels of the $C^{3} \Pi_{u}$ electronic state. This first of all relates to the level with $v^{\star}=4$ and explains its anomalous population density.

The above mentioned regular trends in the intensity growth with increasing amount of argon added, revealed for the $4 \rightarrow 3$ and $3 \rightarrow 2$ lines, correlates very well with the simultaneous increase of the Lewis-Rayleigh glow (Fig. 5). By taking into account the close correspondence of a nitrogen molecule and an argon atom in size, the increase in glow intensity caused by substituting a portion of nitrogen for argon cannot be attributed to an increased probability of recombination of atomic nitrogen, which involves a "third body". This observed feature is likely to be caused only by the higher concentration of nitrogen atoms in the gas phase. Thus, taken together, all the above 
considered facts prove convincingly that the dilution of nitrogen with argon leads to an increase in concentration of atomic nitrogen in the gas phase. This effect becomes more significant with an increasing argon fraction in the gas mixture.

It is also seen from the Fig. 5 that an increase of the argon partial pressure is accompanied by an increase of the intensity of lines of the fourth positive system as compared to a nitrogen plasma. Since the transition of argon to a metastable state is most likely to proceed via an electronic state with excitation energies of no less than $13 \mathrm{eV}$, one can conclude that the increased intensity of lines of this system, which are attributed to transitions from the $\mathrm{D}^{3} \Sigma_{\mathrm{u}}{ }^{+}$state with $12.98 \mathrm{eV}$ energy (for $v^{`}=0$ ), is caused by increased population density of the above mentioned states of molecular nitrogen, because of nonelastic collisions of the latter with argon occurring in the excited states considered. It is known that the potential well of the $\mathrm{D}^{3} \Sigma_{\mathrm{u}}^{+}$state is fairly shallow and the predissociation in this state already occurs at $v^{\prime}>1$ [19]. Consequently, the transition of molecular nitrogen to the $\mathrm{D}^{3} \Sigma_{\mathrm{u}}^{+}$state increases the probability of predissociation and therefore the concentration of atomic nitrogen in ${ }^{4} \mathrm{~S}$ and ${ }^{2} \mathrm{P}$ states.

The long lived argon atoms in metastable states have a considerable influence on the length of the plasma column which extends along the reaction chamber downstream from the RF discharge generation region. Total pressure in the reactor does not affect this area under the conditions studied. Also, it was found that the Lewis-Reyleigh glow intensity stays approximately the same along the plasma column section studied. This shows the constancy of the concentration of atomic nitrogen because of the lower rate of recombination in an argon-nitrogen mixture as compared to a pure nitrogen plasma.

Thus, it was experimentally found that the addition of argon to nitrogen, which was passed through the discharge generation region, leads to a significant increase in the atomic nitrogen concentration because of selective excitation of molecular nitrogen by argon atoms in metastable states.

This conclusion agrees with the results of an experimental study by emission spectroscopy of distribution of some excited particles along the model reaction chamber (Fig. 1) in a $\mathrm{SiH}_{4}-\mathrm{N}_{2}-\mathrm{Ar}$ system. In these experiments nitrogen was introduced from the top of the reactor and a silane-argon mixture ( $4 \% \mathrm{SiH}_{4}$ in $\left.\mathrm{Ar}\right)$ - from the side. The results obtained are shown in Fig. 8 . It should be noted that the lowest possible flow rate of nitrogen (about $1 \mathrm{sccm}$ ) was used in these experiments,

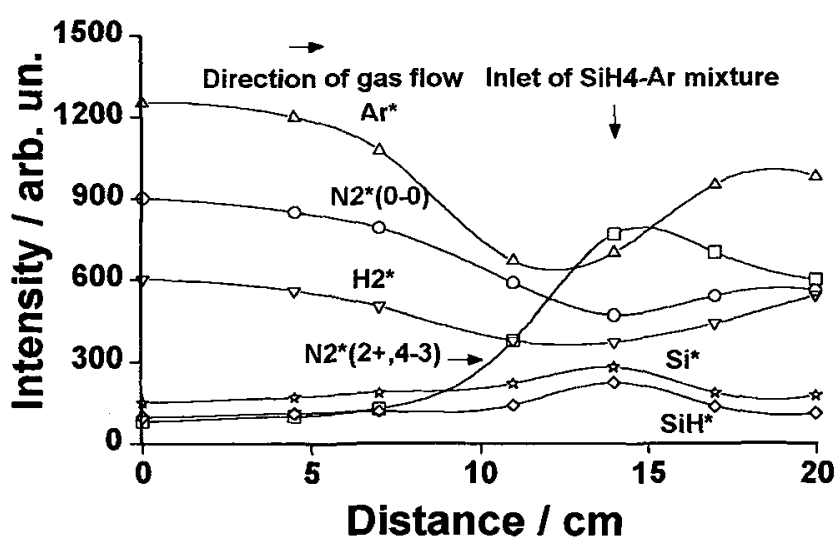

Figure 8: Variations of intensities of selected emission lines with the distance from the discharge generation region along the reactor. because of a considerable increase in the film growth rate on the inner surface of the reactor with an increase of the nitrogen flow rate, and this leads to an inaccuracy of optical measurements. The emission lines corresponding to $\mathrm{Ar}$ and silicon containing species were present in the spectra obtained from an area of the afterglow above the inlet of the $\mathrm{SiH}_{4}-\mathrm{Ar}$ mixture because counterdiffusion of these species was significant under these conditions. However, when the flow rate of nitrogen was approximately equal to that 
usually used for the deposition of $\mathrm{SiN}_{\mathrm{x}} \mathrm{H}_{\mathrm{y}}$ films (not less than $40 \mathrm{sccm}$ ) these lines disappeared in the spectra.

The fall off in the intensities of lines attributed to transitions of $\mathrm{Ar}^{*}, \mathrm{~N}_{2}{ }^{*}(0-0)$ and $\mathrm{H}_{2}{ }^{*}$ (see Fig. 8) and a simultaneous small increase in the intensities of lines corresponding to $\mathrm{SiH}^{*}$ and $\mathrm{Si}^{*}$ radicals in the spectra obtained from the area, where the $\mathrm{SiH}_{4}-\mathrm{Ar}$ mixture was introduced, can be explained by the highest concentration of the silane-argon mixture in this area. This leads to quenching of the emission of the particles with high excitation energies because of their interaction with unexcited particles which have been introduced [20]. It is interesting to consider the dependence for the $4 \rightarrow 3$ line belonging to the second positive system of nitrogen. As one can see the curve corresponding to the $4 \rightarrow 3$ line has a maximum in the area discussed, probably due to the highest concentration of argon in metastable states. In accordance with the conclusion, already



Figure 9: Dedendence of thickness and refractive index of the deposited film on the distance from the discharge generation region along the reactor.

relatively low values of refractive index. It was shown [21] that a decrease of $\mathrm{Si} / \mathrm{N}$ ratio for $\mathrm{SiN}_{\mathrm{x}} \mathrm{H}_{\mathrm{y}}$ films leads to a decrease of their refractive index. Therefore, the results indicate that in this area of the reactor there is the highest concentration of atomic nitrogen formed by direct participation of argon in metastable states. Also it seems likely that the $\mathrm{SiH}_{4} / \mathrm{N}_{\text {at }}$ ratio determines the $\mathrm{Si} / \mathrm{N}$ ratio in the deposited films, and consequently their main properties.

Thus, the results of an emission spectroscopic study show that argon in metastable electronic excited states must play an important role during RPECVD of silicon nitride films in the $\mathrm{SiH}_{4}-\mathrm{N}_{2}-$ Ar system, providing a high concentration of atomic nitrogen necessary for the film formation. It would be interesting to demonstrate this conclusion by a direct experimental study of RPECVD of $\mathrm{SiN}_{x} \mathrm{H}_{y}$ films in the $\mathrm{SiH}_{4}-\mathrm{N}_{2}$-Ar system.

\section{A STUDY OF INFLUENCE OF THE DILUTION OF NITROGEN WITH ARGON ON RPECVD OF SiN $_{X} H_{Y}$ FILMS.}

A detailed description of the reaction system with inductive coupling of the RF power (1.76 Mhz) used in this study has been published previously [22]. A schematic diagram of the reactor is shown in Fig. 1. Briefly, the glow discharge was generated with a RF coil positioned in the upper part of a quartz reactor (diameter $6 \mathrm{~cm}$ ). The flow of nitrogen diluted with argon, both electronic grade, was introduced from the top of the reactor and then directed into the discharge region for excitation of 
the $\mathrm{N}_{2}$ and Ar. A silane-argon mixture ( $4 \%$ of $\mathrm{SiH}_{4}$ ) was introduced downstream from the discharge generation region through the injection nozzle positioned $12 \mathrm{~cm}$ above the substrate. Film thickness and refractive index of deposited layers were measured using an ellipsometer (LEM-3M). The number densities of $\mathrm{SiH}_{\mathrm{i}}$ and $\mathrm{NH}_{\mathrm{j}}$ groups of the deposited films were calculated from FTIR spectra using the method given by Lanford and Rand [23].



Figure 10: Dependence of film growth rate and refractive index with $\mathrm{Q}_{\mathrm{Ar}} / \mathrm{Q}_{\mathrm{N} 2}$ ratio

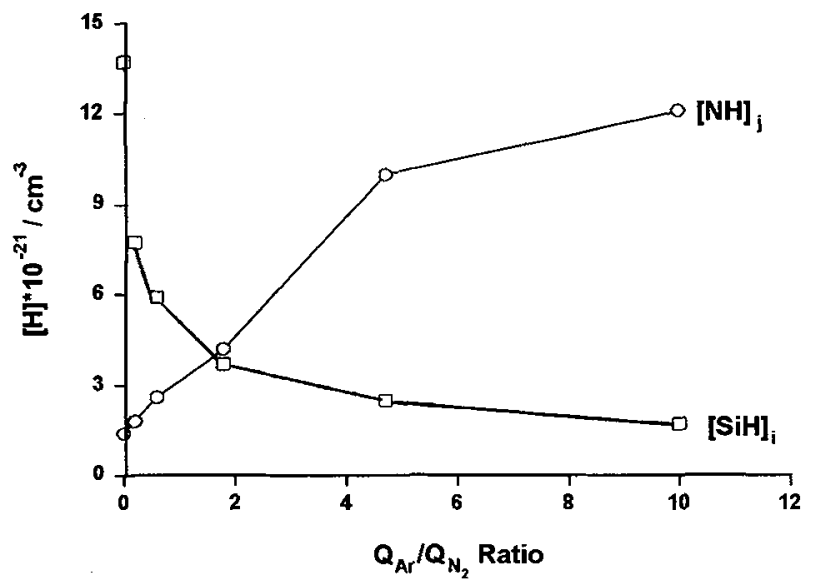

Figure 11: Dependence of content of $\mathrm{SiH}_{\mathrm{i}}$ and $\mathrm{NH}_{\mathrm{j}}$ groups as a function of $\mathrm{Q}_{\mathrm{Ar}} / \mathrm{Q}_{\mathrm{N} 2}$ ratio.
Fig. 10 shows the dependence of growth rate of silicon nitride films on the $\mathrm{Q}_{\mathrm{Ar}} / \mathrm{Q}_{\mathrm{N} 2}$ ratio in the gas flow passed through the discharge generation region. As can be seen there is a gradual decrease of growth rate with dilution of nitrogen with argon. Taking into account that the growth rate of silicon nitride films in PECVD based on using of silane is determined by the concentration of silicon containing radicals [24], formed as a result of dissociation of $\mathrm{SiH}_{4}$ in the plasma, this fall off in growth rate can be explained by a decrease of their concentrations with dilution of nitrogen with argon because of, as already mentioned, the lower electron concentration in the argon-nitrogen RF discharge under research conditions.

The results of measurements of refractive index of as-deposited films demonstrate a gradual decrease of refractive index with an increase of the argon fraction in the argon-nitrogen mixture passed through the discharge region (see Fig. 10). An FTIR study of deposited films did not show any evidences of uncontrollable doping with oxygen. Thus, a decrease of

refractive index of $\mathrm{SiN}_{\mathrm{x}} \mathrm{H}_{\mathrm{y}}$ films probably corresponds to an increase of the nitrogen content in the deposited films with the addition of argon. This agrees with the results of emission spectroscopy considered above. Hence, in spite of a decrease of the relative amount of nitrogen introduced into 
the reaction system, deposited $\mathrm{SiN}_{\mathrm{x}} \mathrm{H}_{\mathrm{y}}$ films become more "nitrogen-rich" because of enhancement of the process of atomic nitrogen formation by selective direct excitation of molecular nitrogen by argon atoms in metastable electronic states.

The analysis of the FTIR spectra showed that apart from the main absorption band related to the stretching mode of the Si-N matrix there is a very weak peak at $3310-3360 \mathrm{~cm}^{-1}$ and another one with a maximum in the region $2140-2180 \mathrm{~cm}^{-1}$, corresponding to the $\mathrm{NH}_{\mathrm{j}}$ stretching modes and $\mathrm{SiH}_{\mathrm{i}}$ stretching modes respectively. A detailed discussion and interpretation of these two peaks has been given elsewhere [6]. From the intensities of these peaks the bonded hydrogen content in the deposited films was calculated. Figure 11 shows that as the $\mathrm{Q}_{\mathrm{Ar}} / \mathrm{Q}_{\mathrm{N} 2}$ ratio increases there is a considerable decrease in the concentration of hydrogen bonded with silicon. At the same time the content of hydrogen bonded with nitrogen is increased by about one order of magnitude. This also confirms an increase in the nitrogen content in the deposited films with an increase of dilution of nitrogen with argon.

It should be noted that silicon nitrides films with refractive index of about 2.05 and good dielectric properties were deposited in several experiments when only argon was introduced from the top of the reactor and then passed through the discharge generation region, and a silane-argon mixture $(6 \mathrm{sccm})$ together with nitrogen $(40 \mathrm{sccm})$ was introduced downstream from the plasma generation region. The presence of charged particles in the area of mixing of the reagents was not specially controlled, but the afterglow zone did not reach this area. Thus, these experiments can be considered as an evidence of possible practical realisation of remote PECVD with an improved control of the process parameters.

\section{CONCLUSION}

The experimental results and considerations presented in this paper show that the main advantage of the remote PECVD processes is protection of the semiconductor (or growing film) surface from degradation due to bombardment by energetic particles during deposition. Remote PECVD processes provide less interdependence for some process parameters, and so they can be more readily controlled. However, completely overcoming this problem for any low pressure RPECVD is impossible. The problem, concerning unpredictable formation of different reacting species in the plasma phase, which cause many parallel reaction pathways, can be mainly solved when the penetration of charged particles downstream from the plasma generation region in the direction of the inlet(s) of precursors is stopped, and neutral particles with definite energies, which in the ideal case must coincide with the values of energy required for the formation of reacting species, are used for the selective excitation. Atoms of noble gases as well as molecules of other gases, in metastable long-lived electronic states, are likely to be successfully used as neutral particles for transferring energy from the plasma region. The example considered of the use of argon atoms in metastable states for the selective excitation of nitrogen molecules with formation of atomic nitrogen necessary for the deposition of different nitride films has shown the possibility of the practical realisation of the type of improved remote PECVD discussed. The experimental results presented in this paper should be considered as only the first step in the long path of development of controllable plasma synthesis. It is clear, that many problems such as those concerning the generation of electrons due to recombination of excited neutral particles via Penning type interaction and diffusion of active species along the reactor still have to be solved. However, the end of this path looks promising. 


\section{Acknowledgements}

The author would like to thank Professor M.L. Hitchman, with whom RPECVD with capacitive coupling of RF power has been studied at the University of Strathclyde, for useful discussions, and my colleagues from St-Petersburg State Technical University for hard work in the field of development of RPECVD with inductive coupling.

\section{References}

[1] Hess D.W. and Graves D.B. in "Chemical Vapour Deposition - Principle and Applications" (ed. Hitchman M.L. and Jensen K.F.) Academic Press, London 1993, 385-435.

[2] Reif R. and Kern W. in "Thin Film Process II ( ed. Vossen J.L. and Kern W.) Academic Press, London 1991, 525-564.

[3] Lucovsky G., Tsu D.V., Rudder R.A., Markunas R.J. in "Thin Film Process II ( ed. Vossen J.L. and Kern W.) Academic Press, London 1991, 565-617.

[4] Gupta M., Rathi V.K., Thangaraj R. et al. Thin Solid Films 204(1991) 77-105.

[5] Helix M.J., Voidyanathan K.V., Streetman B.G. et al. Thin Solid Films 55(1978)143-148.

[6] Alexandrov S.E., Hitchman M.L., Shamlian S.H. Adv. Mat. Opt. Electron. 2(1993) 301-312.

[7] Hattangady S.V., Fountain G.G., Rudder R.A., Markunas R.J. J. Vac. Sci. Tchnol.7(1989) 570-575.

[8] Alexandrov S.E., Hitchman M.L., Shamlian S.H. J. Mat. Chem. 49(1994) 1843-1847.

[9] Kampas F.J. in "Semiconductors and Semimetals" 21A(1984) 153-177.

[10] Smith D.L. in "Plasma technology in Manufacturing of VLSI" (ed. Braun D. and Ainsprooc N.) Mir, Moscow, 1987, 245-264.

[11] Hollahan J.R., Bell A.T. eds. "Techniques and Applications of Plasma Chemistry", John Wiley, New York 1974.

[12] Tsu D.V., Parsons G.N. and Lucovsky G. J. Vac. Sci. Technol. A6(1988) 1849-1854.

[13] Parikh R.A., Hattangady S.V., Posthill J.B. et al MRS Symp. Proc. 102(1988) 275-280.

[14] Alexandrov S.E., Kovalgin A.Y., Rybnikov A.Y. Rus. J. Appl Chem. 66Pt1(1993) 20182023.

[15] Bayers K.D., Kistiakowsky G.B. J. Chem. Phys. 32(1960) 992-1000.

[16] Nemetz V.M., Petrov A.A., Soloviev A.A. eds. "Spectral Analysis of Inorganic Gases", Khimia, Leningrad, 1988, 240 p.

[17] Radtzig A.A. in "Physical Constants: Handbook" (ed. Grigoriev I.S. et al.) Energoatomizdat, Moscow 1991, 794-859.

[18] Huber K.P., Herzberg G. Molecular Spectra and Molecular Structure v. 4 Constants of diatomic Molecules, Van Nostrand Reimond, New York 1979, 716p

[19] Cortwright D.C. Phys. Rev. 16(1977) 1013-1040.

[20] Scheller G.R., Gottscho R.A. Graves D.B. et al J. Appl. Phys. 64(1988) 598-606.

[21] Claasen W.A.P., Valkenburg W.G.J.N., Habraken F.H.P.M., Tamminga Y. J.Electrochem. Soc. 130(1983) 2419-2423.

[22] Alexandrov S.E., Kovalgin A.Y. J. de Physique III, 2(1992) 1421-1429.

[23] Lanford W.A., Rand M.J. J. Appl. Phys. 49(1978) 2473-2477.

[24] Alexandrov S.E., Kovalgin A.Y. Accepted for publication in Rus. J. Appl. Chem.(1995). 